\title{
Fractal analysis of the economic sustainability of enterprise
}

\author{
Andriy Matviychuk ${ }^{1, *}$, Oleksandr Novoseletskyy $^{2}$, Serhii Vashchaiev $^{1}$, Halyna Velykoivanenko $^{1}$, and Igor Zubenko ${ }^{2}$ \\ ${ }^{1}$ Kyiv National Economic University named after Vadym Hetman, Department of Economics and Mathematical Modeling, \\ 54/1, Peremogy Ave., Kyiv, 03680, Ukraine \\ ${ }^{2}$ National university of Ostroh academy, Department of Economics and Mathematical Modelling and Information Technologies, \\ 2, Seminarska Str., Ostroh, 35800, Ukraine
}

\begin{abstract}
The article deals with the method of calculating the fractal analysis, the time series of economic sustainability of the industrial enterprise on the trend-resistant sustainability were investigated by estimating the depth of the long-term memory of the time series and constructing a phase portrait. According to the approach used, the "depth of the long memory" is estimated in terms of fuzzy sets. The approach to the estimation of the index of economic stability is developed, based on the methods of forming an integrated indicator consisting of an assessment of such subsystems as the industrial and technical, financial-economic and subsystem of main parameters of the market environment. These helps to estimate the economic stability of the enterprise in the conditions of incomplete information from purpose of making effective management decisions. Combination of techniques for the formation of an integral index and a fractal analysis of the assessment of its trend stability showed an effective result, which was confirmed by the experiments.
\end{abstract}

\section{Introduction}

Industrial enterprises in a modern economy are often characterized by nonlinear behaviour. It is necessary to solve the problem of identification and rapid response of the financial and production system of the enterprise to the influence of destabilizing factors. One of the directions of the solution of this problem is the use of a system of economic and mathematical models of evaluation and taking into account the economic stability of the enterprise, which will allow to diagnose the current state of the economic system (from the standpoint of dynamics and taking into account the risk) and timely use mechanisms that return the system to equilibrium. It is necessary to estimate the level of economic stability from the position of dynamics using the tools of nonlinear dynamics and fractal analysis. Based on these methods, the Hurst index and the level of long-term memory of the time series are calculated, as well as its trend-stability is established. So, the purpose of research is investigation of economic sustainability of an enterprise by analysing the stability of the trends of its time series using the tools of fractal analysis.

\section{Study Summary}

The method of sequential $R / S$ analysis is presented in [1]. The basis of this technique is a fractal analysis of time series. Let's consider it in more detail using the notation of the time series $Z_{\tau}=z_{1}, z_{2}, \ldots, z_{\tau}, \tau=3,4, \ldots, n$, for each of which the current average is calculated $\bar{z}_{\tau}=$ $\frac{1}{\tau} \sum_{i=1}^{\tau} z_{i}$. Next for each $Z_{\tau}, \tau=3,4, \ldots, n$, we calculate the accumulated deviation for segments of its length $\tau$ :
$X_{\tau, t}=\sum_{i=1}^{t}\left(z-\bar{z}_{\tau}\right), t=\overline{1, \tau}$. After that we calculate the difference between the maximum and minimum accumulated deviations $R=R(\tau)=\max _{1 \leq t \leq \tau}\left(X_{\tau, t}\right)-$ $\min _{1 \leq t \leq \tau}\left(X_{\tau, t}\right)$, which is called "range $R$ ". The next step is to rate $R / S$ by adjusting it to a standard deviation of the time series segment $Z_{\tau}, 3 \leq \tau \leq n$ [2].

The Hurst index $H=H(\tau)$ is determined from the equation $R / S=(\alpha \cdot \tau)^{H}$. Logarithmizing both parts of this equation and assuming, respectively [1], that $\alpha=1 / 2$, we obtain the values of Cartesian coordinates $\left(x_{\tau} ; y_{\tau}\right)$ of points in $H$-path, the ordinate of which

$$
y_{\tau}=\frac{\log \left({ }^{R(\tau)} / S(\tau)\right)}{\log (\tau / 2)},
$$

and abscissa

$$
x_{\tau}=\log (\tau), \quad \tau=3,4, \ldots, n .
$$

The output of the algorithm of $R / S$-analysis is also $R / S$ trajectory, represented in logarithmic coordinates by a sequence of points, abscissa in which $x_{\tau}=\log (\tau)$, and ordinate $y_{\tau}=\log (R(\tau) / S(\tau))$. By connecting the neighbouring points with a segment $\left(x_{\tau} ; y_{\tau}\right)$ and $\left(x_{\tau+1} ; y_{\tau+1}\right), \quad \tau=3,4, \ldots, n-1$, we obtain graphic mapping of the $R / S$-trajectory in the logarithmic coordinates.

The initial stage of the fractal analysis of the time series $Z=Z\left(z_{i}\right), i=\overline{1, n}$ is the formation of the family $S(Z)=\left\{Z^{r}\right\}, r=1,2, \ldots, m$. The time series of this family is obtained by extracting the element $z_{1}^{r-1}$ in the series $Z^{r-1}$. The level of the index $\mathrm{r}$, which reaches the

\footnotetext{
* Corresponding author: editor@nfmte.com
} 
maximum value at the point of trend change in its $R / S$ trajectory, determines the value of $m$. The output timeline is assigned a zero value of the $r$ index [1].

Let's consider the essence of economic sustainability as a system characteristic of an industrial enterprise [3], the time series of which we will examine.

Economic sustainability reflects the ability of the system to maintain an equilibrium state during its operation, to freely manoeuvre with technologies, resources, etc. in the event of the effect of destabilizing external and internal factors, adapt and fulfil the stated purpose in conditions of permissible level of risk for further effective development. That is, the quantitative indicators of the assessment of the level of stability should characterize the economic status of the industrial enterprise, as well as reflect the possibilities and nature of its further development.

\section{Economic sustainability}

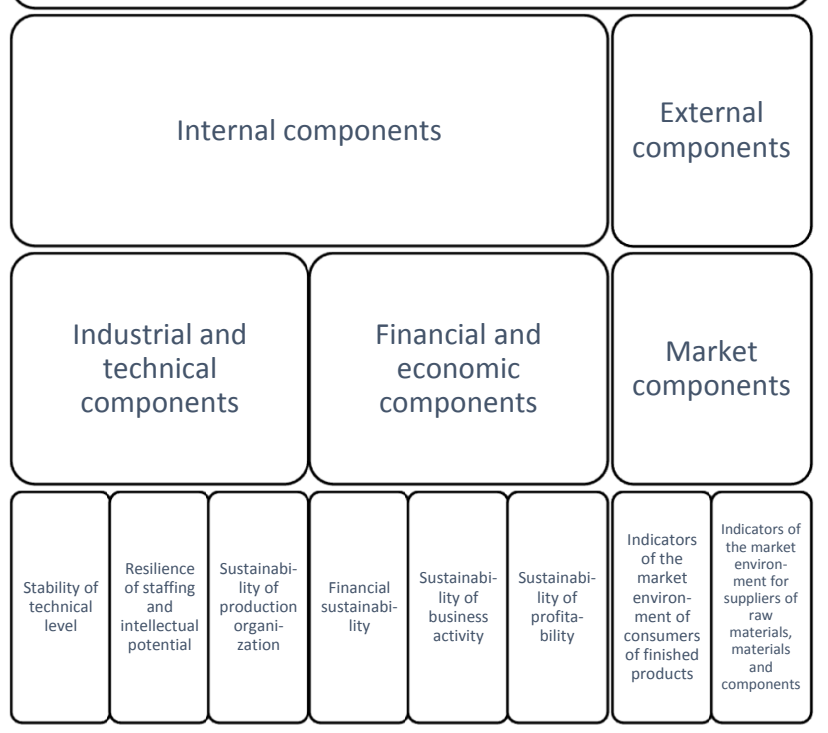

Fig. 1. Dominant hierarchy of estimation of economic stability of the enterprise.

The hierarchy analysis method involves decomposing the problem into separate components, ensuring its structuring and simplification with the construction of hierarchies containing different criteria. The relative advantage of various quantitative and qualitative detailed criteria is determined separately for each indicator of the hierarchical structure from the point of view of the element, which is directly at the highest level of the hierarchy.

Using the hierarchy analysis method an algorithm for assessing the economic sustainability of an enterprise will consisting of the following steps:

Step 1. Formation of a multilevel hierarchical structure, containing an integrated index of economic stability at the upper level, on the following - partial criteria, etc. at the lowest level of the hierarchy there are detailed indicators. We propose to highlight the next indicators:

- indicators that characterize the industrial and technical component of enterprise sustainability $\tilde{S}_{1}$. This is the ratio of the residual value of fixed assets in the currency of the enterprise balance sheet, the coefficient of depreciation of fixed assets, the coefficient of renewal of fixed assets, the coefficient of labour output and return on assets, etc. The choice of these indicators is due to the fact that they allow you to see the level of material, personnel and intellectual potential and the industrial and technical state of the enterprise.

- indicators that characterize the financial and economic component of enterprise sustainability $\tilde{S}_{2}$. These indicators include: coefficient of equity concentration, independence coefficient (autonomy), rapid liquidity ratio, coefficient of manoeuvrability of equity, ratio of own and borrowed funds, coefficient of turnover of equity capital, turnover ratio of material working capital, asset mobility coefficient, return on equity, sales and profitability of the core business. The choice of these indicators in the aggregate is due to the fact that they reflect the financial status, the state of business activity and profitability of the enterprise;

- indicators characterizing the market environment $\tilde{S}_{3}$. This is the coefficient of firm sustainability in the consumer market and in the supplier market. Their choice was due to the fact that the position of the company in these markets determines its economic status.

Step 2. Construction of matrices of pairwise comparisons of elements of the hierarchical structure, which are at each level of the hierarchy (in addition to the integrated one) in terms of the criterion of a directly higher level.

Step 3. Calculation of the vector of weight coefficients of the detailed indicators of the level of stability of the enterprise, located at the lowest level of the hierarchical structure. That is, the quantitative values of the weight coefficients of the detailed indicators are calculated and their rationing is carried out. The obtained indicators $k_{i}, i=1, \ldots, n$ must satisfy, in particular, the condition $\sum_{i=1}^{n} k_{i}=1, k_{i} \geq 0, i=\overline{1, n}$.

Step 4. Calculation of the integral index of economic sustainability. To calculate the quantitative assessment of the level of economic stability use the following formula of the integral indicator:

$$
\begin{gathered}
S_{C}=\sum_{m=1}^{n} \alpha_{m} \tilde{S}_{m} \\
\sum_{m=1}^{n} \alpha_{m}=1,0 \leq \alpha_{m} \leq 1, m=\overline{1, n}
\end{gathered}
$$

The normalized value of partial criteria for economic sustainability of the enterprise $\tilde{S}_{m}$ includes industrial and technical components $\tilde{S}_{1}$, financial and economic components $\tilde{S}_{2}$ and market environment $\tilde{S}_{3}$ [4].

The sustainability level of each subsystem of the economic system including the specific gravity of each of the selected detailed indicators (criteria) $\widetilde{K}_{i}$ calculated as follows

$$
S_{m}=-1+\prod_{i=1}^{n}\left(1+\widetilde{K}_{i}\right)^{k_{i}}, m=1, \ldots, 3, \sum_{i=1}^{n} k_{i}=1 .
$$


The calculated indicators $S_{m}$ should be normalized $\tilde{S}_{m}$.

Step 5. Determination of the dynamics of the level of economic sustainability and the nature of the development of the economy of the industrial enterprise. To do this, we will use instrumentation of nonlinear dynamics, namely, non-linear models of $R / S$-analysis to determine the Hurst index, the availability of long-term memory and the assessment of its depth, as well as trend stability, which will characterize the importance of economic sustainability of the enterprise for several periods of its further functioning.

In Figures 2-4 there are presented the graphical representations of the time series of index of economic sustainability of the industrial enterprise PJSC Consumers-Sklo-Zorya (Ukraine) [5] and the fragment of the $R / S$ - and $H$-trajectories obtained as a result of the application of the $R / S$-analysis algorithm to this time series.

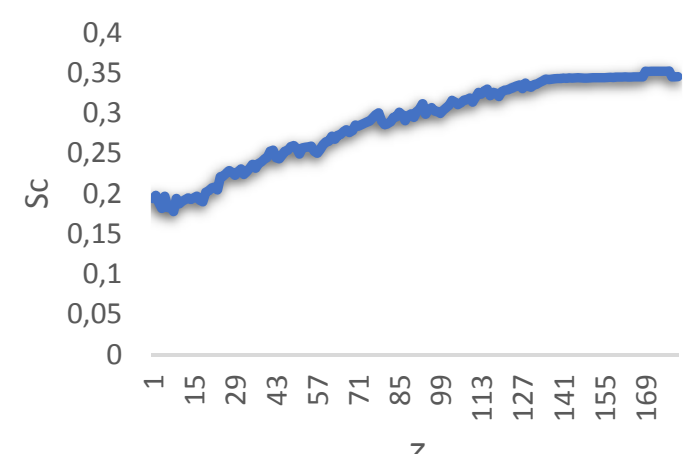

Fig. 2. The time series of index of economic sustainability.

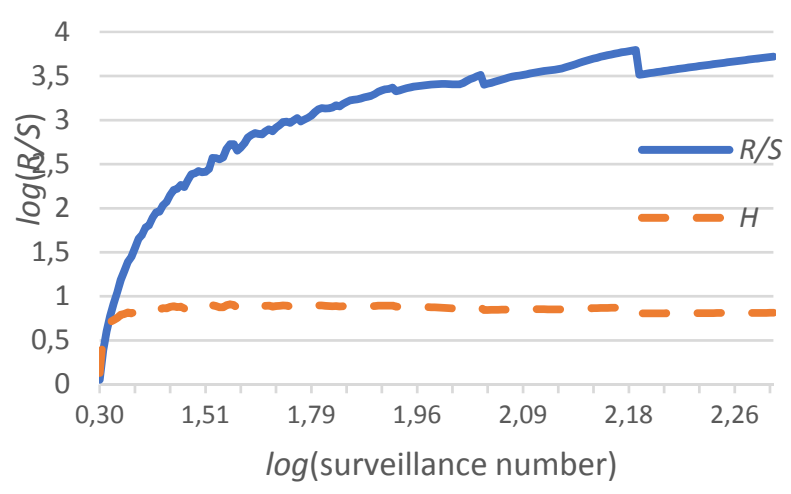

Fig. 3. $R / S$ - and $H$-trajectories of the time series of economic sustainability of enterprise.

The Fig. 4 shows us that the point $\tau=4$ is in the area of white noise and at this point there is a breakdown to the black noise area (the value of $H(5)=0,66$ ), which allows preliminary estimate the depth of the memory in this area of the studied time series $Z_{4}$ by number 4 . Changing the trend of the $R / S$-trajectory at the point $\tau=4$, followed by the transition of the $H$-trajectory into the black noise zone, allows us to estimate the depth of long-term memory by the number 4 . The initial time-series of sustainability has a weak trend-resistance. However, further the series is trend-resistant, which is confirmed by its presence in the vicinity of black noise.

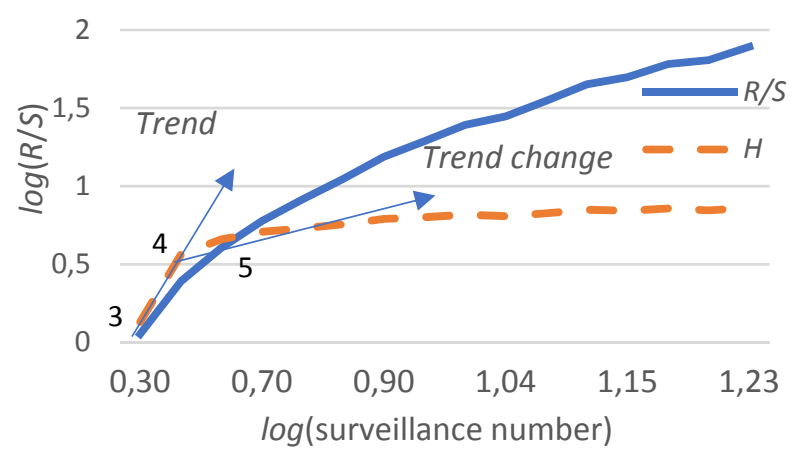

Fig. 4. A fragment of the R/S- and H-trajectories for time series of economic sustainability of enterprise.

Time series of the economic system are not random variables in pure form, the distribution of which probability is subject to a uniform, normal or other known law. Such series have a memory effect and they are called persistent or trend-resistant [6]. Preservation of the trend (probable) for the short-term period may be due to an increase (decrease) in such a series for a limited period. The specified trend stability in some sense is the opposite of short-term "Markov" memory, and we are talking about a time series with memory, in which older events have a tangible impact.

We propose to use the algorithm [7] for estimating the "depth of long-term memory" of the whole time series of economic sustainability and presenting it in the form of a fuzzy set consisting of next steps:

Step 1. Formation on the basis of the time series of index of economic sustainability of the enterprise of $Z$ family $\quad S(Z)=\left\{Z^{r}\right\}, Z^{r}=\left\langle z_{i}^{r}\right\rangle, i=1,2, \ldots, n_{r}$, $r=1,2, \ldots, m$, consisting of $m$ time series $Z^{r}$, where by the index $i$ are marked elements of a $r$-series obtained from the $(r-1)$ time series $Z^{r-1}$ by removing its first element $z_{1}^{r-1}$. Here $m$ is defined as the largest value of the index $r$ such that the series $Z^{m}=\left\langle z_{i}^{m}\right\rangle, i=1,2, \ldots, n_{m}$ still have a point of trend substitution in its $R / S$-trajectory; the output $Z$-series also belongs to the family $S(Z)$, in which it is assigned the index value $r=1$.

Step 2. It is carried out a consistent $R / S$-analysis of time series of economic stability of the family $S(Z)$. The result of the second step is to transform data to form a fuzzy set of values of the depth of memory of the time series.

Let for each time series $Z^{r}=\left(z_{i}^{r}\right)$, $i=1,2, \ldots, n_{r}, r=\overline{1, m}$, as a result of applying to it a sequential $R / S$-analysis algorithm, built $R / S$-trajectory and $H$-trajectory, which determine the number of the point $l_{r}$, in which the trend was changed, that is $l_{r}$ the number $i=$ $l_{r}$ of the first point, which is "higher" than the white noise area, in which the $H$-trajectory received a negative gain, and the $R / S$-trajectory changed the trend.

Enter the following notation: $N(l)$ - the number of all the time series stability $Z^{r}$ of the family $S(Z)$, each with a point number of the trend change $l_{r}$ is equal to the number $l ; l^{0}=\min _{1 \leq r \leq m} l_{r} ; L^{0}=\max _{1 \leq r \leq m} l_{r} ; m=\sum_{l=l^{0}}^{L^{0}} N(l)$; 
$d(l)=\frac{N(l)}{m}-$ the result of such series in the family $S(Z)$, each with loss of memory occurred at a depth of $l$; $L(Z)=\{l\}$ - the range of point numbers trend change in the ranks of the family $S(Z) ; L(Z)=\{l, \mu(l)\}, l \in L(Z)$ - fuzzy set "depth of memory" [8,9] for a time series $Z$ as a whole, $\mu(l)$ - membership function to the "depth $l$ " of the fuzzy set $L(Z)$. Values $\mu(l)$ are proportional to the numbers $d(l), l \in L(Z)$, at the output of step 2 they are obtained by a special normalization of the values of shares $d(l)$ so that $\mu(l)<1$ for any $l \in L(Z)$.

For the time series of index of economic sustainability $Z$ of the industrial enterprise PJSC Consumers-SkloZorya [5], the result of calculations of fuzzy sets of depth of memory of time series is presented in Table 1.

Table 1. Intermediate results of calculations of fuzzy sets of depth of memory of time series $Z$.

\begin{tabular}{|c|c|c|c|}
\hline$l$ & $N(l)$ & $d(l)$ & $\mu(l)$ \\
\hline 4 & 9 & 0,06 & 0,18 \\
\hline 5 & 15 & 0,10 & 0,30 \\
\hline 6 & 35 & 0,23 & 0,69 \\
\hline 7 & 44 & 0,29 & 0,87 \\
\hline 8 & 19 & 0,12 & 0,38 \\
\hline 9 & 18 & 0,12 & 0,36 \\
\hline 10 & 9 & 0,06 & 0,23 \\
\hline 11 & 8 & 0,05 & 0,21 \\
\hline
\end{tabular}

The values of the elements $\mu(l)$ of the last line are calculated as follows. First, they found the maximum share $d\left(l^{*}\right)=\max _{l \in L(\tilde{Z})} d(l)$ (in Table 1 value $\left.d^{*}=0,29\right)$ and its corresponding depth $l^{*}\left(d^{*}(l)=l^{*}\right.$, value $\left.l^{*}=7\right)$. Next, for this depth $l^{*}$ the meaning of the membership function $\mu^{*}=\mu\left(l^{*}\right)$ was expertly identified. Then for the remaining items $l \in L(Z)$ the corresponding value were calculated by the formula $\mu(l)=\frac{d(l)}{d\left(l^{*}\right)} \cdot \mu\left(l^{*}\right)$.

Step 3. By way of pairwise union of elements $N(l)$ and $\mu(l)$, it is formed the fuzzy set of "depths of memory" of the time series of economic stability in general. In our case, it is:

$M(Z)=\left\{\begin{array}{l}(4 ; 0,18),(5 ; 0,30),(6 ; 0,69),(7 ; 0,87), \\ (8 ; 0,38),(9 ; 0,36),(10 ; 0,23),(11 ; 0,16)\end{array}\right\}$.

For clarity, Fig.5 shows a graphical representation of the depths of memory time series economic sustainability of the enterprise.

The result obtained indicates that the memory depth of a particular time series is not a fixed number; its value varies along the studied time series, that is, for each of its segments it is different. For example, as can be seen from Table 1, for the time series of economic sustainability numerical values of depth memory ranges on a segment of a natural $4,5, \ldots ., 11$.

Detection of the depth of long-term memory should serve as ground for constructing a predictive model, which may consider all the essential factors that determine the presence of this memory. In the context of the prediction problem, it is useful to note the basic position of the decomposition analysis [10] of time series. In accordance with this provision, in the general case, the time series can be divided into 4 components: trend, cyclic component, seasonal variation and irregular or final component. In this case, the cyclic component, if it exists, can be a carrier of sufficiently valuable information to make a forecast. In an arsenal of modern methods of prediction of time series, such an approach as visualizing their phase portraits becomes of great importance.

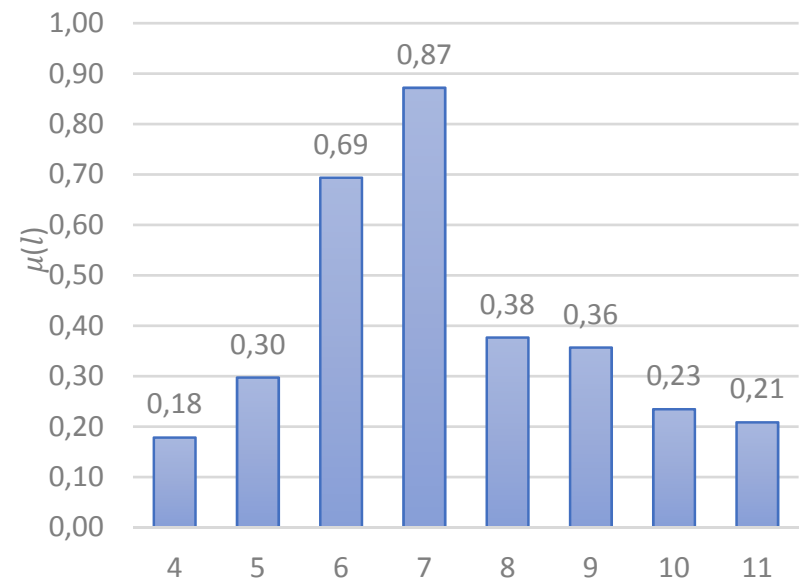

Fig. 5. A graphical representation of a fuzzy set memory depth time series of economic sustainability.

As you know, when you build a phase portrait for a particular time series is fundamentally important question about its dimension $\rho$. This dimension must be no less than the dimension of the attractor of the studied series. In turn, the dimension of the attractor can be estimated with a fairly acceptable accuracy by using the fractal dimension. The latter is calculated by the formula $C=2-H$. Since for the time series considered in this paper the value is given $H \in(0 ; 1)$, we obtain an estimate $C<2$. Thus, for our purposes, there are reasons to use a phase space $F(Z)$ of dimension $\rho=2$ [1].

Such a phase trajectory of the time series of economic sustainability is presented in Fig. 6.

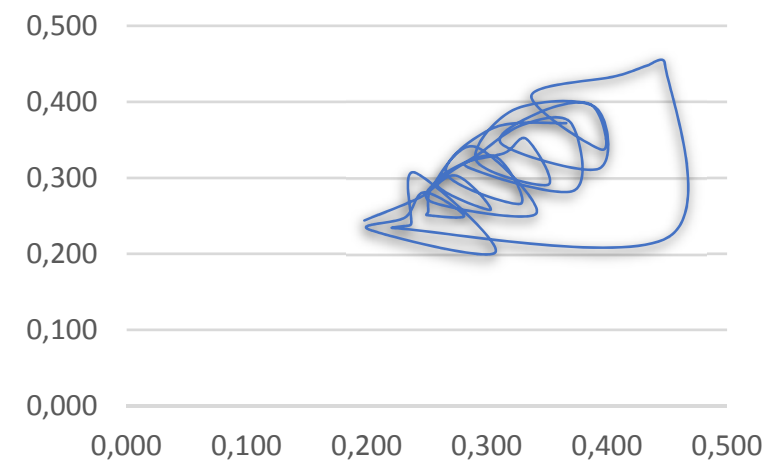

Fig. 6. A phase portrait of a time series in a two-dimensional phase space.

For its construction we used calculated data from Table 2.

Consider this phase portrait in the form of a trajectory, namely, in the form of a sequence of points in which each adjacent pair is connected by a segment or curve. In this 
trajectory we also select its segments, which are called quasicycles [11]. The definition of quasicycle is close to the concept of a cycle. The difference between these two concepts is that the initial and final quasicycle points do not have to match. The end point of a quasicycle is determined by its occurrence in the baseline of the initial point, while self-propelling the initial and final links of the quasicycle is allowed, if this leads to a maximum approximation of the initial and final points. In reality there are such series of economic processes in which phase portraits contain such pairs of non-neighbouring time points, in which the coordinates in the phase space actually coincide. The presence of such pairs of points actually destroys the cyclic structure of phase trajectories.

Table 2. Output for abscissa and ordinates.

\begin{tabular}{|c|c|c|c|c|c|}
\hline Period & $\begin{array}{c}\text { Indicator } \\
\text { value } \boldsymbol{z}_{\boldsymbol{i}}\end{array}$ & $\begin{array}{c}\text { Indicator } \\
\text { value } \boldsymbol{z}_{\boldsymbol{i} \mathbf{+}}\end{array}$ & Period & $\begin{array}{c}\text { Indicator } \\
\text { value } \boldsymbol{z}_{\boldsymbol{i}}\end{array}$ & $\begin{array}{c}\text { Indicator } \\
\text { value } \boldsymbol{z}_{\boldsymbol{i}+\mathbf{1}}\end{array}$ \\
\hline 1 & 0,198 & 0,244 & 25 & 0,448 & 0,451 \\
\hline 2 & 0,244 & 0,274 & 26 & 0,451 & 0,222 \\
\hline 3 & 0,274 & 0,303 & 27 & 0,222 & 0,234 \\
\hline 4 & 0,303 & 0,258 & 28 & 0,234 & 0,237 \\
\hline 5 & 0,258 & 0,292 & 29 & 0,237 & 0,240 \\
\hline 6 & 0,292 & 0,330 & 30 & 0,240 & 0,307 \\
\hline 7 & 0,330 & 0,370 & 31 & 0,307 & 0,202 \\
\hline 8 & 0,370 & 0,372 & 32 & 0,202 & 0,232 \\
\hline 9 & 0,372 & 0,283 & 33 & 0,232 & 0,248 \\
\hline 10 & 0,283 & 0,315 & 34 & 0,248 & 0,281 \\
\hline 11 & 0,315 & 0,333 & 35 & 0,281 & 0,250 \\
\hline 12 & 0,333 & 0,351 & 36 & 0,250 & 0,251 \\
\hline 13 & 0,351 & 0,291 & 37 & 0,251 & 0,255 \\
\hline 14 & 0,291 & 0,323 & 38 & 0,255 & 0,290 \\
\hline 15 & 0,323 & 0,390 & 39 & 0,290 & 0,341 \\
\hline 16 & 0,390 & 0,393 & 40 & 0,341 & 0,253 \\
\hline 17 & 0,393 & 0,312 & 41 & 0,253 & 0,269 \\
\hline 18 & 0,312 & 0,345 & 42 & 0,269 & 0,307 \\
\hline 19 & 0,345 & 0,387 & 43 & 0,307 & 0,328 \\
\hline 20 & 0,387 & 0,396 & 44 & 0,328 & 0,266 \\
\hline 21 & 0,396 & 0,337 & 45 & 0,266 & 0,306 \\
\hline 22 & 0,337 & 0,408 & 46 & 0,306 & 0,366 \\
\hline 23 & 0,408 & 0,434 & 47 & 0,366 & 0,372 \\
\hline 24 & 0,434 & 0,448 & 48 & 0,372 & \\
\hline & & & & & \\
\hline
\end{tabular}

So, overall, the trajectory of the phase portrait (Fig.6) the time series of economic stability consists of eleventh quasicycles $C_{r}, r=1, \ldots, 11$. Fig. 7-10 shows some fragments of these quasicycles.

The dimensions $L_{r}$ of these quasicycles are presented in Table 3.

Table 3. Quasicycles and their dimension are the result of a phase portrait for the time series of economic stability.

\begin{tabular}{|c|c|c|c|c|c|c|c|c|c|c|c|}
\hline$C_{r}$ & $C_{1}$ & $C_{2}$ & $C_{3}$ & $C_{4}$ & $C_{5}$ & $C_{6}$ & $C_{7}$ & $C_{8}$ & $C_{9}$ & $C_{10}$ & $C_{11}$ \\
\hline$L_{r}$ & 4 & 5 & 4 & 4 & 4 & 5 & 5 & 4 & 5 & 4 & 4 \\
\hline
\end{tabular}

Compare the depth of memory of the investigated time series, which is represented by a fuzzy set (5) with the quantifiers of the quasicycles, which are reflected in the second row of Table 3. From this comparison it follows that the presence of long-term memory in the analysed time series, along with other factors, is also due to the cyclical component of this time series.

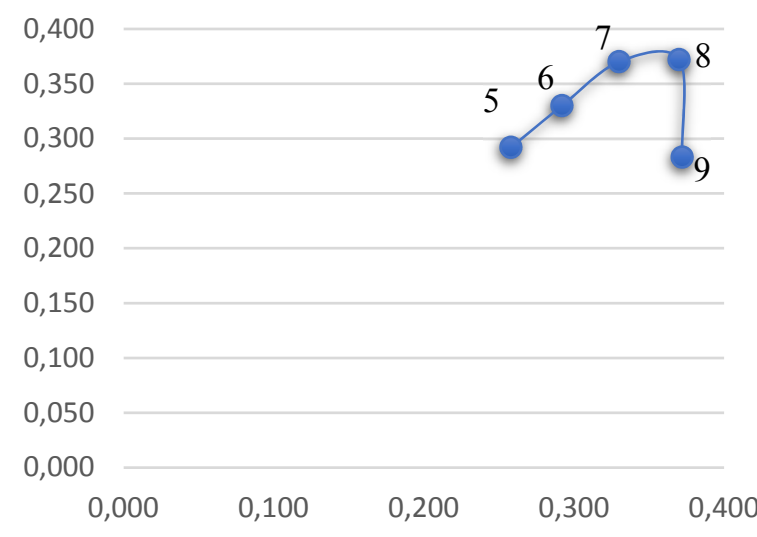

Fig. 7. The second quasicycle of the output timeline $Z$.

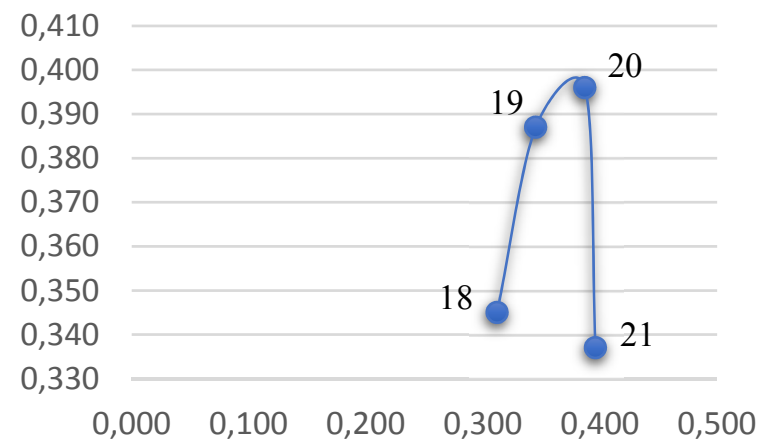

Fig. 8. The fifth quasicycle of the output timeline $Z$.

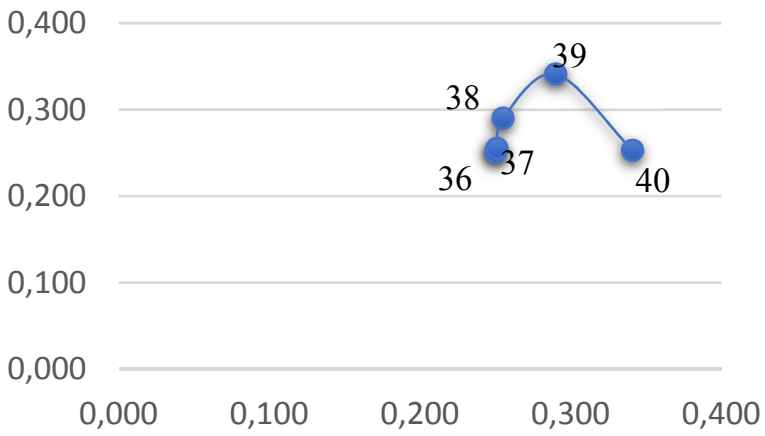

Fig. 9. The ninth quasicycle of the output timeline $Z$.

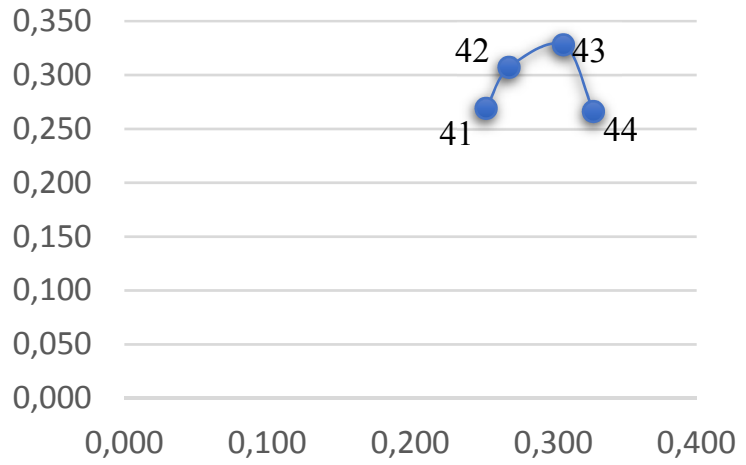

Fig. 10. The tenth quasicycle of the output timeline $Z$. 


\section{Conclusions}

The algorithm for calculating the depth of long-term memory developed on the basis of fractal analysis showed that the depth of a particular time series is not a fixed number but is changing. One of the reasons for this is the cyclical time-series component, based on which we can talk about the creation of predictive models.

Implementation of the considered methodology at the industrial enterprise showed that the time series of economic sustainability of the enterprise is trendresistant. This means that the level of economic stability will remain within the trend during a certain period of further enterprise operation due to available assets and reasonable administrative policy of management.

\section{References}

1. Maksishko, N.K., Perepelica, V.A.: About two approaches to $\mathrm{R} / \mathrm{S}$ analysis of time series. Visnik of the Volodymyr Dahl East Ukrainian national university. 5, 134 (2005)

2. Soloviev, V., Solovieva, V., Solovieva, K.: Comparative analysis of the dynamics of the stock market of Ukraine using fractal measures of complexity. Bulletin of Cherkasy University. Series of Economic Sciences. 33, 246 (2012)

3. Maksishko, N.K.: Estimation of system characteristics of economic dynamics based on the results of complex fractal analysis. Herald of Zaporizhzhya National University. 2, 10 (2011)

4. Novoselytskyy, O.: Methodology for calculating the economic stability of the enterprise in the light of risk. Formation of market relations in Ukraine. 11, 78 (2007)

5. SMIDA. PJSC Consumers-Sklo-Zorya (Ukraine). https://smida.gov.ua/db/participant/22555135 (2010). Accessed 21 Mar 2019

6. Peters, E.: Chaos and Order in the Capital Markets: A New View of Cycles, Prices, and Market Volatility. John Wiley \& Sons, New York (1996)

7. Maksishko, N.K.: About applying an algorithm of sequential $\mathrm{R} / \mathrm{S}$ analysis to obtain a fuzzy estimate of the depth of memory of the time series. Herald of Lviv University. 516 (2006)

8. Maksishko, N.K.: Modeling of economy by methods of discrete nonlinear dynamics. Polygraph, Zaporizhzhya (2009)

9. Maksishko, N.K., Perepelica, V.A.: Analysis and forecasting of the evolution of economic systems. Polygraph, Zaporizhzhya (2006)

10. Holden, K., Peel, D.A., Thompson, J.L.: Economic Forecasting: An Introduction. Press Syndicate of the University of Cambridge, Cambridge (1990)

11. Melikhov, E., Ebzeeva, N.: Mathematical modelling of economic time series based on phase portraits. Paper presented at the International Conference "Mathematics. Economy. Education", Rostov-onDon, May 29 - June 52005 